\title{
Política pública-violencia versus derecho a la ciudad en el centro histórico de Puebla, México
}

\author{
Public politics-violence versus the right to the city \\ in the historic center of Puebla, Mexico
}

\begin{abstract}
Marisa Higuera Cortés•
Virginia Cabrera Becerra*

Lilia Varinia Catalina López Vargas ^••

\section{Resumen}

Este trabajo se orienta a develar la dupla política pública-violencia, en el centro histórico de Puebla, México, en su papel instrumental a intereses de rentabilidad, banalizando las reivindicaciones del derecho a la ciudad. La dupla política pública-violencia se analiza en momentos actuales, considerados relevantes, pero la breve referencia histórica realizada muestra su presencia en la fundación de la ciudad. El camino metodológico se trazó desde el marco teórico epistémico de la complejidad, que llevó a considerar como clave para comprender las particularidades locales el entrelazamiento entre la indagación teórica, el contexto nacional y el histórico. La revisión histórica, bibliográfica, así como la observación y aplicación de entrevistas exploratorias apuntalaron la confirmación del choque enunciado como

Abstract

This work is aimed at revealing the violence in the public politics, in the historic center of Puebla, its instrumental role to profitability interests, trivializing the claims to the right to the city. The pair public politics-violence is analyzed at present times, considered relevant, but the brief historical reference shows its presence in the foundation of the city. The methodological path was drawn from the epistemic theoretical framework of complexity that led us to consider as a key to understanding local particularities, the interaction between theoretical inquiry, national and historical context. The historical and bibliographic review, as well as the observation and application of exploratory interviews, underpinned the confirmation of the shock stated as the direction of the investigation.
\end{abstract} directriz de la investigación.

Palabras clave: política pública, violencia, derecho a la ciudad, ciudadanía.
Keywords: right to the city, public politics, violence, citizenship.

\footnotetext{
-Estudiante de la Maestría en Territorio,Turismo y Patrimonio, del Instituto de Ciencias Sociales y Humanidades “Alfonso Vélez Pliego" de la Benemérita Universidad Autónoma de Puebla. Licenciada en Psicología por la Facultad de Psicología de la Benemérita Universidad Autónoma de Puebla. mary_hico@hotmail. com Orcid: 0000-0003-49/8-0770.

- Doctora en Urbanismo por la Universidad Nacional Autónoma de México. Profesora-investigadora en el Instituto de Ciencias Sociales y Humanidades “Alfonso Vélez Pliego" de la Benemérita Universidad Autónoma de Puebla. virginia@urbe.com.mx_ ORCID:0000-0002-6I54-9174.

- Doctora en Ciudad, Territorio y Patrimonio por el Instituto de Urbanística de la Universidad de Valladolid. Profesora-investigadora en la Facultad de Arquitectura del Instituto de Ciencias Sociales y Humanidades “Alfonso Vélez Pliego" de la Benemérita Universidad Autónoma de Puebla. variva35@ yahoo.com.mx Orcid: 0000-000I-7844-219X.

Fecha de recepción: 2 de octubre de 2019. Fecha de aceptación: 30 de noviembre de 2020.
} 


\section{Introducción}

Con el surgimiento, en Inglaterra y Estados Unidos, de la fase neoliberal del capitalismo hacia la posguerra se produjeron transformaciones sin precedentes en el mundo. $\mathrm{Al}$ expandirse en Latinoamérica y particularmente en México, a finales de la década de los ochenta, se derivan una multiplicidad de consecuencias económicas, sociales y ambientales que constituyen herencia enraizada en nuestros territorios.

Una de las expresiones más funestas del modelo neoliberal es la desigualdad, si bien de raíces históricas más profundas, que en México nos remiten a la época de la Conquista y al periodo Prehispánico, no hay duda de que con el modelo neoliberal se ha reproducido, ampliado y profundizado, adquiriendo magnitudes que han sido el caldo de cultivo de la violencia, la corrupción y la impunidad. Los territorios, las ciudades y las comunidades rurales han sufrido los embates del modelo neoliberal, en los cuales el despojo de tierras y la destrucción de nichos de vida han propiciado resistencias y violencias.

El Estado ha ejercido un papel instrumental, allanando los caminos para avanzar en los procesos de despojo a través de un amplio arco de acciones, entre las cuales el uso de la violencia ha sido recurrente. A la violencia del Estado se ha sumado la violencia derivada del narcotráfico y la herencia del sistema patriarcal: la exclusión, y la desigualdad de clase, raza y género. Tres gruesas raíces: Estado, narcotráfico, patriarcado, que nutren la violencia en los territorios, las ciudades y las comunidades rurales, contrastan con los anhelos de inclusión, igualdad y pluralidad que la sociedad expresa constantemente en foros de opinión pública y especializada y en la vida social cotidiana. Anhelos y principios que en el ámbito de algunas disciplinas se han fundido en el concepto del "derecho a la ciudad", el cual de entrada nos 
remite a la ciudad, una particular forma socioterritorial de la gama de expresiones del territorio, de donde el concepto en sí mismo encierra, paradójicamente, exclusión.

Las reflexiones presentes emanan del objetivo de aproximarse al entramado conformado por la política pública, la violencia y la ciudad, desde dos miradas: la teórica y la empírica; destacando la posibilidad de ruptura a través de la ciudadanía. Para el logro del objetivo trazado mantenemos como base epistémico-metodológica el pensamiento de la complejidad, que nos orienta en la identificación de procesos estratégicos para desentrañar el entramado que interesa estudiar (Morin, 2001, 2006; García, 2008). Debido a la comprensión de fenómenos socioterritoriales como fenómenos complejos, éstos deben ser analizados a partir de los elementos que se vinculan y entrelazan desde distintos niveles. Este marco se expresa en el análisis de condicionantes históricas y globales, como necesarios para comprender la situación existente a nivel nacional y estatal, dado que permite ubicar y explicar los rasgos particulares del caso estudiado. La estructura y análisis que se presenta obedece a esta conceptualización.

En el primer apartado se desarrolla una breve exploración teórica a partir de las aportaciones de algunos autores acerca de los conceptos: derecho a la ciudad y ciudadanía; este último remite a la posibilidad de ruptura y reconducción, sustentada en la participación de la sociedad, en la formulación y aplicación de la política pública. En el segundo apartado se elaboran algunas reflexiones acerca de la incidencia de la globalización y el modelo neoliberal en la modernización de las ciudades, con la consiguiente profundización de la desigualdad como sustrato nutriente de la violencia y la inseguridad, con objeto de significar la categoría de política pública como instrumento coadyuvante en la organización del territorio y de las ciudades para beneficio de determinados grupos de la sociedad vinculados al 
poder político y económico. En el tercer apartado se analizan las condiciones que presenta este entramado en la ciudad de Puebla, México, con dos ejemplos relevantes de política pública. En busca de una mejor comprensión, el análisis de dichos ejemplos es antecedido por dos rubros: en un primer momento se ubica a Puebla en las condiciones de violencia del país, para después reflexionar acerca de la construcción de la ciudad desde la violencia contenida en las condiciones de segregación construidas históricamente. Con base en este recorrido se delinean, a manera de conclusiones, algunas reflexiones respecto de la trama enunciada.

\section{Derecho a la ciudad y ciudadanía}

El concepto de "derecho a la ciudad" elaborado por Lefebvre desde los años sesenta del siglo pasado, es retomado en los últimos años por autores como Jordi Borja, David Harvey, Manuel Delgado, entre otros. Con diferencias en la forma de entenderlo, abordarlo y problematizarlo, algunos lo observan como una suma de derechos en la ciudad y otros rescatan el derecho a la ciudad como una reivindicación política para la construcción de una ciudad de derechos.

El derecho a la ciudad, en el sentido de Lefebvre, implica "el derecho a vivir en una sociedad donde todas las personas tienen la misma libertad para satisfacer sus propios deseos y donde todos reciben apoyo para hacerlo" (Marcuse, 2010: 92). En este sentido, no se trata sólo de una suma de los derechos individuales, sino de destacar que se exige el derecho colectivo de que se garanticen tales derechos.

Retomando la idea de la ciudad, de raigambre marxista, como el lugar de la reproducción de la fuerza de trabajo que desarrolla Lefebvre, se observa un énfasis en sus dos vertientes: la producción y el consumo. Lefebvre señala lo urbano como central en la formación de las fuerzas productivas por su propia capacidad de generar prácticas y 
representaciones sociales. Para Lefebvre lo urbano es una totalidad que se compone tanto por el modo de vida como por la base morfológica y material y, por lo tanto, el derecho a la ciudad contempla tanto el derecho a la apropiación como a la participación.

Por su parte, David Harvey lleva el derecho a la ciudad a un sentido más colectivo. Para él, este derecho no implica sólo la garantía de derechos individuales, sino también un sentido de colectividad. Según Harvey, "es un derecho a cambiar y reinventar la ciudad de acuerdo con nuestros deseos" (Harvey, 2005: 20). Este autor aporta la idea de que la reivindicación del derecho a la ciudad será aquella que responda a la acumulación por desposesión con movimientos urbanos; se trata de un derecho colectivo de "quienes facilitan la reproducción de la vida cotidiana" (Harvey, 2012: 201) en la ciudad y la construyen materialmente. A lo señalado por tales autores, agregamos que habría que explicitar el derecho a la ciudad de quienes se encargan de la reproducción de la fuerza de trabajo y de las condiciones de reproducción de la vida; de los trabajos de reproducción y de cuidados que tradicionalmente han realizado las mujeres. El derecho a la ciudad es, por tanto, el ejercicio del poder colectivo sobre el proceso de construcción de la ciudad.

Para Jordi Borja la ciudad "es a la vez condición y expresión de la ciudadanía, de los derechos ciudadanos" (Borja, 2002: 39), en tanto los derechos garantizados o negados se manifiestan en la ciudad. Dicha relación establecida por el autor responde a la idea de que ya no se tratan solamente de derechos humanos, sino de derechos que reconocen el carácter político del ciudadano. Sin embargo, tampoco se trata de descartar los derechos humanos, sino de asumir que el ciudadano tiene el derecho a exigir que a través de las políticas públicas se hagan efectivos sus derechos (Borja, 2013). 
Según el autor, los derechos de los ciudadanos se manifiestan en el espacio público y, a la vez, son politizados en él; por ello cuando no exista un espacio público eficaz, no existirán tampoco derechos garantizados. Rescatar el papel del espacio público como sitio de expresión de los derechos significa privilegiar el espacio destinado a manifestar la diversidad social y el interés de los sujetos de derechos por decidir el rumbo de la ciudad. Entre los derechos reivindicados, Borja menciona el trabajo, la vivienda, los espacios y servicios públicos, las luchas populares y conquistas urbanas, y el acceso a la información entre las instituciones y la ciudadanía. Estando de acuerdo en las atribuciones y posibilidades del espacio público que Borja enfatiza, hemos de dejar asentada la exclusión que contiene respecto del espacio doméstico, en el cual todavía transcurre mayoritariamente la vida de las mujeres que integran una parte igualmente importante de la ciudad. Por lo que agregaríamos que el derecho a la ciudad pasa necesariamente por el espacio doméstico como parte esencial de la dinámica urbana.

Abundaremos ahora en el tipo de actor que reivindica el derecho a la ciudad: la ciudadanía. Para ello Miguel Pérez define la ciudadanía como "un sistema regulatorio por el cual las comunidades políticas administran sus diferencias internas" (2015: 11). Con base en ello, el autor problematiza y discute sobre la formación de la ciudadanía urbana y el proyecto de derecho a la ciudad propuesto por Lefebvre. El autor se remite a la conformación de la ciudadanía moderna identificándola como resultado de la repartición de derechos en un contexto donde las clases sociales "discutían sobre asuntos comunes desde su condición de privados" (Pérez, 2015: 11).

En las condiciones de desigualdad que prevalecen en las sociedades, la ciudadanía moderna se imbrica con el concepto de igualdad, lo que en términos prácticos significa dotar de los mismos derechos a todos los ciudadanos (Pérez, 
2015: 10) y es un concepto central al ejercer y defender los derechos. Las reivindicaciones de los derechos deben ser congruentes con lo anterior, de modo que se tiene que diferenciar los derechos en la ciudad y el derecho a la ciudad. En este sentido, podemos observar una diferencia sustancial entre las formas en que se usa este concepto en las cartas del derecho a la ciudad y el sentido crítico que le dio Henri Lefebvre cuando acuñó el concepto como "un grito, una demanda, una reivindicación” (Marcuse, 2010: 91).

En síntesis, los atributos delineados por los autores mencionados respecto al concepto de derecho a la ciudad son enunciados desde posiciones propias de las culturas hegemónicas. Por ello, afirmamos que su aplicabilidad para nuestro país y particularmente para Puebla debe ser reflexionada a la luz de las condiciones y características específicas.

\section{Para significar la política pública}

Al tiempo que nacía la teoría e ideología neoliberal en el mundo, en México veíamos la crisis del Estado benefactor y sus consecuencias políticas, económicas y sociales. Además, otros factores productivos como la crisis de la rigidez fordista, y económicos como la crisis de los Tratados de Bretton Woods (Salazar, 2005: 28), entre los resultados de dichos tratados estuvieron la creación en 1944 del Fondo Monetario Internacional (FMI) y del Banco Mundial (BM). A estos antecedentes se sumó más recientemente, a finales de los años ochenta, el Consenso de Washington, del que derivaron las políticas económicas de corte neoliberal como puntales de la instauración de gobiernos neoliberales en México.

Momentos clave fueron los sexenios de Miguel de la Madrid (1982-1988), de Carlos Salinas de Gortari (19881994) y posteriormente de Ernesto Zedillo (1994-2000), donde este modelo se consolidó en la estructura de gobierno 
mexicano (Salazar 2005: 31). A esta lista de sexenios, por supuesto, hay que agregar los de Vicente Fox (2000-2006), Felipe Calderón (2006-2012) y Enrique Peña Nieto (20122018) como momentos de declive acelerado del sistema político construido e instrumentalizado por el modelo neoliberal, en donde la trama violencia, política pública y ciudad conformó un flujo tan líquido que penetró en cada intersticio de nuestros territorios. El actual Gobierno federal (2018-2024), liderado por Andrés Manuel López Obrador, ha declarado "el fin de la política neoliberal en México" (López Obrador, 2019). Con ello se deslinda de los anteriores gobiernos y expresa la voluntad transformadora de su gobierno por instalar un modelo que privilegie la política social enfocada en los más desfavorecidos. El conjunto de acciones que se están desplegando en nuestro país son los primeros indicios de la ruta posible que se transitará en pos del anhelo formulado.

La estrategia neoliberal en el caso de México fue, en resumidas cuentas: la superación de los desequilibrios macroeconómicos, el control de la inflación, la renegociación de la deuda externa, la liberalización comercial y financiera, aceleración de la venta de empresas paraestatales y la eliminación de la regulación estatal (Salazar, 2005: 37). Este tipo de política pública resultó en control y estabilización económica a cambio de la dependencia del financiamiento e inversión internacional, especialmente de Estados Unidos, y con costos sociales como la radicalización de la desigualdad social y económica al interior del país. Rescatamos la afirmación de Hasan (2010) de que la promoción del libre mercado por parte de tres instituciones globales: la Organización de las Naciones Unidas (ONU), el Fondo Monetario Internacional (FMI) y la Organización Mundial de Comercio (OMC), incide en el campo inmobiliario y en el desarrollo urbano, por representar actividades económicas lucrativas 
que dinamizan la inversión en el desarrollo inmobiliario, en infraestructura y servicios urbanos.

De donde coincidimos con la afirmación de Ornelas de que la ciudad actual, dentro de la lógica del capitalismo, se sujeta a "la tendencia del capital a elevar la productividad del trabajo mediante la división técnica del trabajo y la socialización de las condiciones generales de la producción y los servicios" (2004: 142). Como señala el autor citado, a nivel territorial esto implica la localización de actividades vinculadas al ejercicio de poder dentro de la ciudad; así como la concentración de las condiciones generales de la producción y las fuerzas de trabajo en los centros urbanos (Ornelas, 2004: 146). Como consecuencia se ha producido una superconcentración de la población en las ciudades que responde al interés y condiciones que establece el capital. Lo que se traduce en una configuración específica del espacio urbano legitimada bajo políticas públicas que el Estado elabora e implementa para facilitar la acumulación del capital.

En síntesis, con el modelo capitalista, particularmente en la actual etapa de globalización y neoliberalismo, la modernización de la ciudad con fines de rentabilidad guía la formulación y aplicación de la política pública. En nuestro país la desigualdad se profundiza, allanando el camino para la creciente violencia e inseguridad que atraviesa los territorios urbanos y rurales. De modo que la ciudad se organizará en función de la competitividad, así, para el caso particular de Puebla, en el periodo gubernamental de Manuel Bartlett (1993-1999) la política pública explicita el objetivo de lograr posicionar a Puebla en los circuitos internacionales del capital. 
La distancia profunda entre la teoría del derecho a la ciudad y la vida cotidiana

En las condiciones mexicanas actuales, en las que el territorio, ciudades y campo padecen condiciones de inseguridad y violencia creciente, es necesario resignificar el derecho a la ciudad, ya que se ha generado desde 2006 una diversificación y expansión territorial de la violencia criminal. Situación que provocó una frontal destrucción de los derechos humanos. Falla fundamental de esta estrategia fue, precisamente, no tener un enfoque en derechos humanos lo que facilitó que se cometieran injusticias y violaciones a los derechos humanos, como detenciones arbitrarias, torturas, desapariciones forzadas, violaciones y homicidios. El derecho a la ciudad ha quedado, entonces, nulificado en la dimensión esencial del derecho a la seguridad y a la vida. Las condiciones del país obligan a resignificar el concepto, dándole centralidad a estos dos rasgos mencionados.

Nos podemos aproximar a la magnitud del problema a través de los datos aportados por López, López y Olvera: entre 2006 y 2013 los desplazamientos internos a causa de la violencia suscitada por el combate al narcotráfico alcanzan los 700,000 habitantes; México está clasificado en muertes por conflicto armado en el año 2014 como tercer país a nivel mundial con un aproximado de 15,000 muertes. Respecto al desplazamiento por despojo como consecuencia de la construcción de presas hidroeléctricas y de actividades extractivas, la autora señala que en el año 2016 fueron 200,000 los desplazados de sus comunidades y para el caso de desplazados por desastres naturales la cifra asciende, entre el año 2008 y 2013, a dos millones de personas (López, López y Olvera, 2018: 25).

Con las acciones que se están desplegando por el actual presidente Andrés Manuel López Obrador que han suscitado debates, adherencias y oposiciones, se observa un 
radical cambio de enfoque al dirigirse a las bases estructurales. Este cambio, como todo proceso histórico, está también permeado de continuidades ancladas a condiciones heredadas; así, la multiplicidad de programas sociales se entremezcla con la "guardia nacional" que implican rupturas y continuidades tanto por su entrelazamiento como por su composición interna.

Nos acercamos, entonces, a las expresiones actuales de la violencia en Puebla, en un momento en que en el país ha adquirido magnitudes que desbordan el imaginario de lo creíble y arrojan por la borda a los derechos humanos y al derecho a la ciudad, en tanto la configuración de territorios y ciudades se encuentra signada por la violencia. Pero hablar de violencia nos puede conducir a dos ideas: la violencia observable a través de los delitos, crímenes y guerras que Zizek llama violencia subjetiva; y la violencia que encontramos en los entramados y configuraciones del sistema social, político y económico, llamado por Zizek violencia sistémica. De la multiplicidad de aspectos que constituyen el problema de la violencia, interesa analizar dos vertientes: la violencia delictiva cotidiana y la violencia anudada a la política pública; esta última se desarrolla a través de dos casos concretos en Puebla.

Respecto de la primera vertiente, los análisis geoestadísticos del INEGI indican que Puebla tiene una tasa del delito por debajo de la media y que, por lo tanto, no es de las ciudades más violentas. Sin embargo, el $31 \%$ de la población ha sido víctima de un delito. Pero, aunque la cifra es alta, no es mayor que en otros estados, pues a nivel nacional la tasa del delito es de 37,017 (INEGI-Envipe, 2017).

Acerca de la percepción de la inseguridad, la Envipe del INEGI refiere que en el año 2017 el 68.1\% de los habitantes del estado de Puebla consideraba que la entidad federativa era insegura, mientras que a nivel nacional el promedio era del 78\% (INEGI-Envipe, 2017). Además, sobre lugares donde nos 
sentimos inseguros, el cajero automático en la vía publica nos parece inseguro al $84.6 \%$ de la población, seguido del banco (76.3\%) y el transporte público (73.6\%) (INEGI-Envipe, 2017). Se habla, en su mayoría, de espacios públicos, por lo que se interpreta que son los lugares públicos los que son percibidos como inseguros y violentos. Situación que encubre una realidad que no puede soslayarse cuando se habla de violencia e inseguridad y que implica la violencia que ocurre en el espacio doméstico, privado, anudada a las condiciones inherentes al sistema patriarcal. Aunque no es el foco de las presentes reflexiones, consideramos importante no invisibilizarlas, pues hablar del derecho a la ciudad implica también expandir las posibilidades de seguridad y desarrollo a los espacios que hacen ciudad desde la cotidianidad del habitar.

Con base en este panorama nos acercamos a las condiciones de la violencia en la ciudad de Puebla como nivel territorial necesario para ubicar y dimensionar lo que ocurre en su núcleo central. Para ello se elaboró la cartografía de delitos en la ciudad a partir de la integración de una base de datos de los delitos publicados por El Sol de Puebla de 2010 a 2016, ubicando los lugares donde se cometieron (véase ilustración 1). 
Ilustración 1. Cartografía de delitos publicados

por El Sol de Puebla
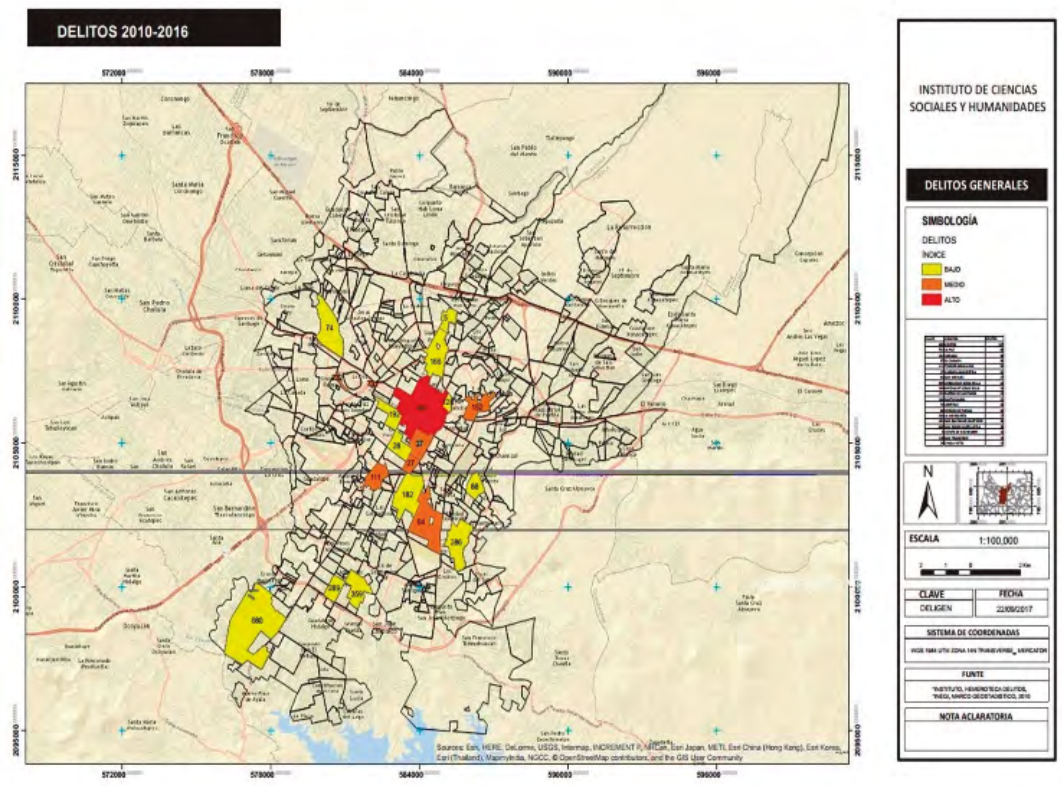

Fuente: elaborado con base en información de Marisa Higuera Cortés, desarrollada para el proyecto "El paisaje cultural construye una ciudad segura”. Dibujo cartográfico: Getsemaní Marín. Mapa base: OpenStreetMap.

Al ubicar los delitos según la colonia donde fueron cometidos, el centro histórico aparece con la mayor concentración de ellos; además de colonias y barrios aledaños. No es casual que sea el centro histórico el espacio donde más delitos se registran pues por su importancia social enfoca la atención de los actores sociales que construyen el centro histórico. Sin embargo, esto no implica que realmente sea el lugar donde más delitos se cometen o donde mayor violencia existe, simplemente se señala que es el lugar donde, desde la opinión pública, se da mayor atención a la violencia delictiva. Éste es un primer hallazgo acerca de la expresión territorial de la 
violencia en Puebla, que contribuyó a su delimitación como área de estudio a partir de su importancia social.

\section{Puebla, configuración territorial signada por la violencia}

Hablar de la violencia en el centro histórico de Puebla conlleva a establecer como punto de partida que la violencia constituye una problemática de densa complejidad por sus múltiples expresiones, causales, efectos y lugares de ocurrencia. Generalmente se constriñe la violencia a su expresión individual o grupal, como el uso de la fuerza física que causa daño e incluso la muerte a las personas o que perjudique sus bienes. Sin embargo, cuando se habla de violencia urbana se limita a la consideración de la ciudad como el lugar, el escenario de los hechos violentos.

Nuestro interés se centra en las expresiones de violencia en su vinculación con el territorio y la ciudad, específicamente interesa mostrar la violencia en su maridaje con la política urbana, vinculación poco explorada desde las disciplinas espaciales, y la implicación de tal anudamiento con el derecho a la ciudad.

En las expresiones múltiples de la violencia hay que considerar que, a nivel subjetivo, existe una elaboración del territorio de la violencia por cada persona; para alguien una calle, una zona, un barrio o una ciudad puede ser violenta, mientras que para otra persona esa misma calle puede no serlo. A la complejidad que estas dimensiones otorgan a la violencia agregamos su permanente movilidad en el transcurso histórico. Baste señalar que el centro histórico de Puebla está marcado por las huellas de la violencia desplegada históricamente y entrelazadas con las formas que actualmente se despliegan en él y por él.

El centro histórico de la ciudad de Puebla ha sido disputado por diversos grupos con interés en apropiarse de su centralidad; que para algunos se traduce en mayor renta- 
bilidad económica y política, y para otros, los habitantes, en el lugar en el que transcurre la lucha cotidiana por la supervivencia. En el centro histórico de Puebla la violencia ha sido constante en su historia. El fenómeno nos remite hasta la fundación de Puebla, la cual fue configurada con una lógica de segregación: el área del asentamiento de los conquistadores y el área indígena, donde los indígenas tenían acceso restringido al asentamiento de aquéllos, salvo para la realización de actividades de servidumbre.

La configuración espacial de la traza urbana fue exclusiva para los conquistadores con la amenaza de que el indio que no cumpliera esto sería azotado. Así, la violencia simbólica se materializa en la configuración del territorio, pues "estas distribuciones jerárquicas del espacio muestran el uso de una violencia simbólica dañina a la libertad de movimiento y de acción" (Salazar, 2005: 11). En consecuencia, esta "situación de desigualdad provocó un sentimiento de injusticia y de agravio moral que se tradujo en actitudes violentas que formaron parte de la vida cotidiana de la población" (Salazar, 2005: 11). Los castigos generalmente conducían a la muerte y se realizaban en espacios públicos para servir de advertencia, para disciplinar a la población en la obediencia.

En el siglo xx la segregación, como forma de exclusión que violenta, se dio con la creación de zonas de tolerancia y la normatividad alrededor de casas de prostitución y cantinas o pulquerías. Durante las primeras décadas del siglo la llamada zona de tolerancia fue destinada para el ejercicio de la prostitución, estableciéndose en el barrio de San Antonio, límite nororiente del centro histórico (véase ilustración 2). Este barrio fue influenciado económica y espacialmente por tal delimitación, pues también desarrolló "los giros comerciales directos como bares, cantinas y pulquerías, además de los locales de abasto y servicios complementarios como misceláneas, fondas, sastrerías, zapaterías y bolerías" (Hernández, 2015: 12). 
Como observamos, el centro histórico ha vivido, en sus diversas etapas, múltiples acciones que lo ubican como un espacio signado por la violencia, que segrega a quienes considera violentos por su condición social, económica o étnica, o bien que es disputado como territorio de grupos que utilizan la violencia como significante social. A través de estos procesos históricos la violencia y la segregación han estado vinculadas.

Se ha construido la idea, desde el discurso oficial, de que el espacio del centro histórico, como patrimonio cultural, se encuentra bajo amenaza y descuido, especialmente por la violencia delictiva, por lo que los sujetos que lo dañan deben ser expulsados. Esta idea sirve de base para implementar políticas y proyectos de segregación y expulsión de la población considerada potencialmente violenta, pero también de la más vulnerable. La política pública desplegada en el centro histórico de Puebla entra en franca contradicción con la idea del derecho a la ciudad, que desde el discurso pregona (Implan, 2019).

En los últimos años la vocación turística del centro histórico, sustentada en su centralidad y atributos estéticos e históricos, ha sido utilizada como argumento para implementar políticas tendentes a intensificar afluencia turística, cultural y comercial por parte de visitantes. Se han traducido en beneficios financieros para ciertos sectores económicos y políticos, mientras que los habitantes de las vecindades son expulsados y los usuarios habituales del centro histórico como estudiantes y trabajadores pasan a segundo plano. A continuación se ilustra lo dicho a través de dos ejemplos que muestran cómo desde políticas públicas urbanas se configuran relaciones de violencia. 


\section{Programa de Descentralización Comercial}

\section{como eufemismo de elitización social}

En Puebla es recurrente la disputa por los espacios públicos en el centro histórico entre ambulantes, comerciantes, empresarios y fuerza pública; en un contexto en el que las exigencias de apropiación de la centralidad con fines de rentabilidad propicia acciones para sacar de las calles al ser marginado y eliminar toda huella de pobreza y no la pobreza en sí. Con las consecuencias de transformación que favorecen la gentrificación del centro de la ciudad, el paisaje urbano y social entra en un proceso de valorización a expensas de sus habitantes y, por supuesto, del propio patrimonio históricamente creado.

Este fenómeno es antecedido por un proceso de evolución de la ciudad con dos escenarios bien identificados: el centro y la periferia (Guerrero, 2005). El periodo de expansión urbana que según algunos autores inició a partir de los años cuarenta se caracteriza por ser un proceso en el que, al tiempo en que se desarrollaba el equipamiento e infraestructura para la periferia destinada a las clases media alta y alta, el centro se convirtió en un escenario propicio para ser ocupado por las clases bajas provenientes de los sectores empobrecidos de la ciudad, pero también de migrantes de zonas rurales y municipios aledaños a la capital. Entre las consecuencias del proyecto de modernización de la ciudad, que en su momento le apostó al desarrollo industrial, se encuentran altos índices de migración, el incremento de la densidad de la población y, por lo tanto, el incremento de las actividades económicas de subsistencia. El aumento para esa época fue de casi cinco veces y la concentración de estas actividades se dio en el centro, sobre todo se trató del comercio ambulante (Guerrero, 2015).

El crecimiento constante del ambulantismo asentado en el centro histórico, en confluencia con las exigencias de reapropiación del espacio por parte de los sectores económica- 
mente rentables, fueron condicionantes para la implementación a partir de 1986 del Programa de Desconcentración Comercial, por parte del Gobierno municipal presidido por Jorge Murad Macluf. Mediante este plan se creó la Central de Abastos y siete mercados de apoyo en áreas periféricas de crecimiento urbano, además de la expulsión de comerciantes del antiguo Mercado La Victoria y ambulantes ubicados en la actual calle 5 de Mayo entre 4 y 8 Poniente (Vélez, 2007: 125), para culminar con el cierre del mercado en 1986. La intención real de apropiación para actores y actividades de mayor rentabilidad se puede descubrir en la formulación de sus objetivos, ya que el programa establece entre éstos: rescatar el centro histórico, promover el turismo, resolver el problema el ambulantismo.

El Mercado La Victoria a mediados del siglo xx se fortaleció como centro de abastecimiento y ventas comerciales formales e informales al interior y fuera de la ciudad, extendiéndose las ventas en las calles y manzanas alrededor de él. Esto era un claro obstáculo, desde la idea de modernización y rentabilidad del suelo, para llevar a cabo la dignificación del centro histórico, pues se concibió que esta actividad tenía que ser desplazada hacia otras zonas y con ello a las personas que, desde sus posibilidades y limitaciones, habían formado un modo de empleo y de vida como ambulantes, pero también como artesanos, practicantes de oficios, cocineras, etcétera (Patiño, 2002: 108).

Como consecuencia se desalentó al traslado de habitantes de la ciudad y zonas aledañas para el suministro de víveres y mercancías en el centro de la ciudad, pero la mayor afectación según Vélez fue hacia los habitantes más pobres del centro histórico (Vélez, 2007: 126), cuyo trabajo dependía de las actividades comerciales en dicho mercado y el nuevo traslado del hogar al trabajo donde habían sido reubicados, implicando mayor costo de tiempo y dinero. A pesar de que el ambulantaje representaba una forma de trabajo conse- 
cuencia de la combinación de la incapacidad para generar la cantidad y calidad de empleos para la población y de una forma de comercio tradicional que se originó en los antiguos mercados prehispánicos, también representaba, por las condiciones materiales y por ser realizada por quienes viven en condiciones de pobreza, un obstáculo para los intereses de modernización que se pretendía en el centro histórico por parte de los grupos económicos y políticos más fuertes (Cabrera, 2008).

En términos generales, el cierre del Mercado La Victoria, sumado a otras intervenciones del Plan, tuvo como principal consecuencia el gradual despoblamiento del centro histórico. Pero lo que esto implica en la sociedad no es sólo la transformación urbana, sino un cambio en la forma en que los habitantes se relacionan con su ciudad, es decir, se trata de condiciones de vida y de trabajo más complejas y desgastantes para el habitante.

La política urbana de desconcentración comercial del centro histórico implicó una fuerte violencia del Estado contra las formas de vida y la vida misma de los habitantes empobrecidos del centro histórico, resultó no sólo en el asesinato de comerciantes sino también en la creación de diversas organizaciones de lucha popular que han continuado hasta la actualidad. A pesar de la política de desalojo del comercio informal del centro histórico y, en específico, del Mercado La Victoria y aun a pesar de haber convertido el antiguo mercado en un centro comercial a cargo de la Fundación Amparo, el comercio informal persiste y el ambulantaje se reapropia constantemente de la zona, resistiendo y enfrentando la violencia instrumentada desde el Gobierno.

Esta situación implica que la zona alrededor del antiguo Mercado La Victoria sea disputada por las organizaciones de ambulantes, por lo que dicho espacio varía de ocupantes según los acuerdos de las organizaciones con el Ayuntamiento del municipio de Puebla. La disputa por dichos 
espacios del centro histórico muestra cotidianamente acciones violentas que visibilizan la dimensión física de la misma y la conformación de un imaginario de la violencia geográficamente localizada.

Con fines de percepción de violencia e inseguridad, se realizó un acercamiento a través de entrevistas aplicadas a 60 habitantes de la ciudad de Puebla, en el año 2016. En las entrevistas, algunos habitantes de la ciudad señalan que entre los lugares considerados como violentos se encuentran: el centro, específicamente las calles 8 y 10 Oriente, el barrio de Analco y el barrio de Santiago. Es en la calle 5 de Mayo, en el tramo comprendido entre las calles 6,8 y 10 Oriente el que es ocupado por vendedores ambulantes. Esta combinación propicia la apropiación del espacio público por comerciantes informales y es percibida por la población como un lugar peligroso, transformándose en un imaginario de violencia.

Estos imaginarios también pueden verse expresados en los mapas mentales elaborados por habitantes de la ciudad de Puebla, como la siguiente ilustración que muestra cómo para un habitante masculino la zona circundante del Mercado La Victoria es un espacio donde existe violencia subjetiva (ilustración 2), y expresa en entrevista que se debe a los delitos que se cometen en la zona, así como de la presencia de los grupos de ambulantes. 
Ilustración 2. Mapa mental de violencia en el centro histórico. Masculino de 24 años. Proyecto: "El paisaje cultural construye una ciudad segura"

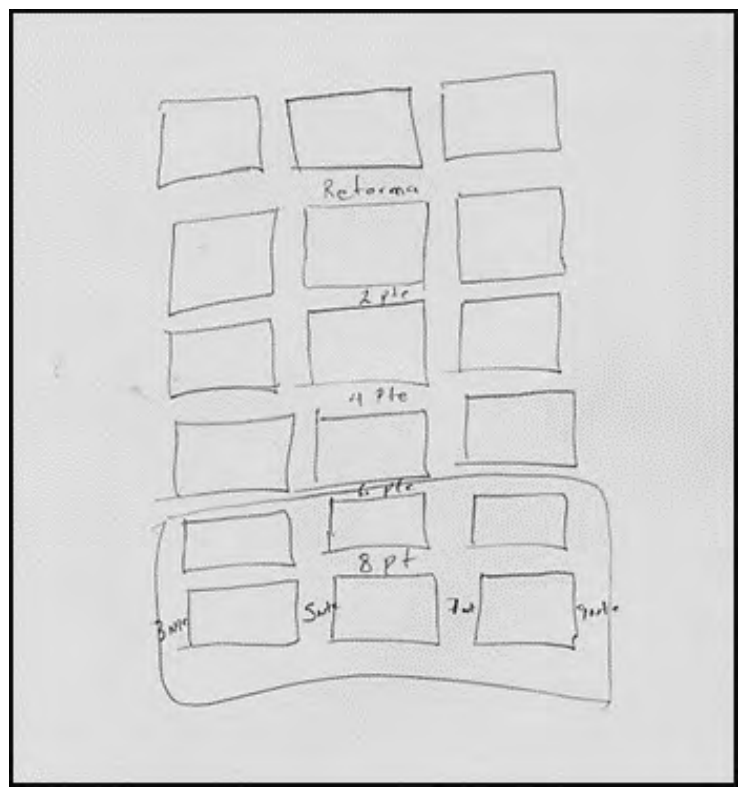

Estos imaginarios son construidos socialmente y se reproducen a través de hechos cotidianos, así como de sucesos extraordinarios, tal como sucedió el día 22 de julio de 2016 durante la alcaldía de Luis Banck Serrato, en la calle 8 Oriente-Poniente y 5 de Mayo, donde una operación de desalojo realizada de manera violenta por parte de la policía contra ambulantes provocó un enfrentamiento entre ellos y alarmó a los cientos de personas que habitan y transitan el centro histórico de la ciudad. Según reportes, el hecho sucedió aproximadamente a las 13:30 horas; sin embargo, tal y como se observó empíricamente en trabajo de campo, el miedo colectivo provocó el cierre de negocios y escuelas, así como el abandono del centro histórico hasta 
el día siguiente. El saldo según noticias periodísticas fue de 15 heridos (Hernández, 22 de julio de 2016).

El desalojo de los ambulantes en dicho espacio es constantemente demandado por la población y por grupos empresariales, quienes argumentan que al ser los ambulantes una modalidad del comercio informal, afecta sus ventas y dan mala imagen al lugar; argumentos tradicionalmente manejados en la disputa recurrente que se establece por la apropiación del espacio público del centro histórico. El ambulantaje continúa siendo un obstáculo para el proyecto de elitización del centro histórico y, sin embargo, no se atienden las causas de la falta de empleo formal, mientras se estigmatiza una forma de comercialización que tradicionalmente se ha realizado sobre las calles. La ubicación de grupos delictivos, desde secuestradores, tratantes de mujeres, carteristas y asaltantes, en la misma zona en la que se concentran los ambulantes actualmente no es casual; aprovechan la desorganización y la concentración de personas para pasar inadvertidos, al tiempo que al confundirse con vendedores sirve para que se legitime el constante acoso y violencia de las autoridades contra los ambulantes.

\section{Paseo del Río San Francisco, política y conflicto}

Constituye el segundo ejemplo a través del cual se muestra la unidad: política pública-violencia, ya que su aplicación desató violencia y represión, para viabilizar la apropiación de suelo e inmuebles históricos, generando un paulatino proceso de recomposición social conocida como gentrificación. El programa denominado Paseo del Río de San Francisco se desarrolló en el área del Río de San Francisco, sitio en donde tuvo lugar la fundación de la ciudad de Puebla en 1531.

Debido a la necesidad de abastecimiento de agua, la primera fundación de la ciudad fue en la orilla oriente del Río San Francisco y posteriormente funcionó como frontera 
física para dividir a la población de conquistadores de la indígena. El Río San Francisco marcó la separación entre la ciudad de Puebla y los barrios indígenas asentados del otro lado del río. Para finales del siglo XIX y principios del xx, las orillas del Río San Francisco fueron aprovechadas para ubicar fábricas y curtidurías debido a la proximidad con el vital líquido. Sin embargo, para mitad del siglo $\mathrm{xx}$ las consecuencias de la alta actividad industrial y los residuos urbanos vertidos en el Río se visibilizaron en la contaminación de éste. En atención al problema, en 1963 las autoridades entubaron el agua del Río y construyeron sobre él una de las vías urbanas más importantes para la ciudad: el Boulevard 5 de Mayo, que además coadyuvaría a la lotificación de terrenos de las zonas norte y sur de la ciudad (Vélez, 2007: 98). Por lo que se propició la urbanización del área y facilitó la movilidad en la ciudad.

En 1993, el Programa Angelópolis implementado por el gobernador Manuel Bartlett Díaz contempló una serie de acciones para el centro histórico, entre las que se encontraba el Programa del Paseo Río San Francisco. El gobierno de Bartlett consideró necesario para el centro histórico el desarrollo de infraestructura para servicios. Bajo esta idea se generó la formación de "un distrito cultural, turístico y de negocios" (Vélez, 2007: 140), integrando la zona de Los Fuertes con el centro histórico. Dicho proyecto apostaba a la promoción de capitales nacionales y extranjeros en un marco de tercerización económica (Cabrera, 2014). Con esa idea se propuso un proyecto de intervención en la zona de Los Fuertes de Loreto y Guadalupe y del lado oriente del Río San Francisco, que incluía la instalación de centros expositores, hoteles, restaurantes, estacionamientos y oficinas. Parte del proyecto del Río San Francisco, ya que la idea original se modificó, se realizó en una zona donde existían viviendas que eran consideradas por quienes elaboraron el proyecto como "edificios que ofrecen un pésimo aspecto al paisaje 
urbano” (Pavón, 1982: 50, citado por Vélez, 2007: 142), pues se trataba de la zona de barrios indígenas y según Vélez (2007), para los creadores del proyecto, la arquitectura y el valor urbano de los barrios indígenas son menores que el de la traza española.

Para sustentar el proyecto se elaboró el Programa Parcial de Desarrollo Urbano, Mejoramiento e Integración del Paseo del Río San Francisco que fue aprobado por el H. Cabildo del municipio de Puebla en agosto de 1993 (Vélez, 2007: 146). En dicho programa se establecen las modificaciones al uso de suelo de la zona correspondiente sólo al Río San Francisco y adyacentes.

Para Vélez, el conflicto central del proyecto no es solamente la transformación urbana y arquitectónica del centro histórico, sino que el mismo proyecto tuvo un impacto en la vida social del lugar, sobre todo para los habitantes de viviendas, vecindades y departamentos. Al considerarse un proyecto de interés público, los habitantes de la zona fueron desalojados y despojados de los inmuebles expropiados haciendo uso de la fuerza pública; mientras que aquellos edificios rentados eran desalojados por los propietarios (Cabrera, 2014: 7).

La comunión de la política pública y la violencia adquiere en estos momentos carta de naturalidad. Sin embargo, antes de llevar a cabo el proyecto, debido a la forma en que se presentó, algunos de los propietarios y vecinos se organizaron y constituyeron una sociedad civil, mientras que otros se concentraron en organizaciones como la Unión de Barrios, el Comité de Inquilinos y la Organización de Colonos Democráticos (Revista Patrimonio Cultural, 1994: 24). Estas organizaciones solicitaron el respeto a la cultura de los barrios, ampararse contra la declaratoria de utilidad pública con la que podían expropiar sus predios y la búsqueda de alternativas para el mejoramiento de las viviendas. Sin embargo, a pesar de la oposición al proyecto por parte 
de los habitantes y de cierto recelo del INAH y del Consejo del Centro Histórico al no obtener información detallada del proyecto, el Gobierno del estado y los encargados del proyecto encontraron el respaldo de empresarios poblanos quienes, en el caso Paseo San Francisco, observaban una buena oportunidad para el desarrollo turístico de Puebla con expectativas de beneficios económicos.

Como anteriormente se mencionó, el lado oriente del Río San Francisco fue asignado, durante los primeros años de la ciudad de Puebla, como lugar exclusivo para los asentamientos indígenas. Para finales del siglo xx esta zona de barrios antiguos como Analco, El Alto y La Luz era ocupada por industrias, vecindades y pequeños talleres de oficios tradicionales; sin embargo, tras la imposición del proyecto Paseo San Francisco a orillas de lo que anteriormente fue el Río San Francisco se ha producido una serie de transformaciones en esta zona que suman al deterioro y expulsión de sus habitantes tradicionales, así como de la pérdida de la riqueza cultural de los barrios tradicionales.

Es visible, a través de la observación de campo, el deterioro de los barrios afectados por el proyecto, como son: el abandono de inmuebles, alta percepción de inseguridad y la mala calidad de servicios e infraestructura para sus habitantes, condiciones que hacen latente la especulación inmobiliaria sobre estos barrios. En parte consecuencia del proyecto Paseo San Francisco sobre los barrios, a pesar de que el área de impacto del proyecto sólo abarca seis manzanas: el área entre Boulevard 5 de Mayo y 12 Norte, y entre la Avenida 2 Oriente y 14 Oriente. Actualmente se encuentran espacios privados, semipúblicos, privados de acceso público, y privados como: una iglesia, una escuela privada, un centro comercial y de convenciones, hoteles y restaurantes de alta categoría, un parque público, oficinas de gobierno y estacionamientos. 
Este conjunto de espacios constituye actualmente el complejo del Paseo San Francisco, donde a pesar de albergar instalaciones de gobierno e, incluso, patrimonio arqueológico, es percibido en general como un gran espacio privado de uso público y con acceso limitado. Sin embargo, esta cualidad de espacio comercial extiende su impacto sobre el Boulevard 5 de Mayo, donde se disponen actualmente más hoteles, comercios y centros comerciales de alto impacto y costo, mercantilizando además al patrimonio para fines meramente turísticos. Por el lado opuesto, del lado de los barrios antiguos podemos observar, además de oficinas de gobierno, casas habitacionales en aparente abandono y deterioro.

\section{Ilustración 3. Área de uso comercial y turístico circundante a Paseo San Francisco}

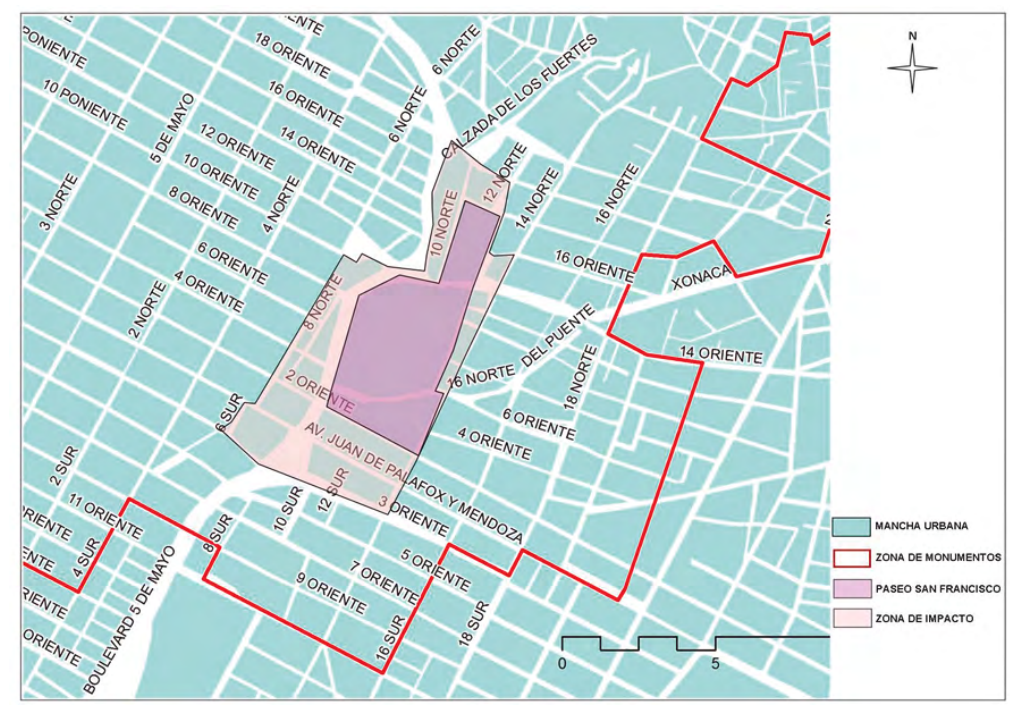

Fuente: elaborado con base en información de Marisa Higuera Cortés, desarrollada para el proyecto "El paisaje cultural construye una ciudad segura”. Mapa base: Víctor Steven Hernández Toscano. 
Contrasta que al acercarse al mercado La Acocota, ubicado en el Barrio de la Luz, persistan comercios populares y vecindades, así como departamentos de bajo costo. Del mismo modo, hay la percepción de un alto nivel de inseguridad, así como constantes denuncias sobre asaltos, robos, acoso sexual y narcomenudeo en la zona. A pesar de la existencia del mercado La Acocota, otras formas de comercio informal han sido perjudicadas, como pequeñas tiendas y comida de calle como las chalupas del parque San Francisco y puestos de zaguán (Meléndez, 2017).

Aunque en un principio el proyecto no tuvo el éxito esperado en términos de inversión, recientemente ha ido consolidándose como un importante centro comercial, obteniendo un incremento en los visitantes y en los locales comerciales ocupados. Esto se logra principalmente por la instalación de oficinas de gobierno en la zona (Meléndez, 2017) y aunque algunos mencionen que este lugar es un espacio privado con uso público, estrictamente hablando se trata de un lugar privatizado que, posteriormente y sólo en cierta medida, se ha abierto al público. Esto es evidente incluso en el propio Parque de San Francisco que se encuentra constantemente vigilado por miembros de seguridad privada y pública, además de la presencia de cámaras de video-vigilancia. Asimismo, la permanencia en este sitio se condiciona a un reglamento expuesto a la vista de los visitantes. El acceso a áreas reconocidas como patrimonio arqueológico es restringido e, incluso, clausurado al público. Ejemplo muy conocido de esta situación es la que se realizó en 2017 por parte del hotel Rosewood de los lavaderos de Almoloya que datan de 1863, ubicados en el área de impacto del proyecto.

En general, observamos que el proyecto Paseo San Francisco fue creado como un centro de desarrollo económico en el centro histórico, enfocado principalmente en el área de turismo y servicios. Su desarrollo coincidió con uno de los momentos clave de la inserción del país en la política econó- 
mica capitalista neoliberal, su implementación violentó los derechos de los habitantes a su ciudad, particularmente los derechos de los habitantes desplazados del lugar. El acceso es controlado por rejas, portones eléctricos y miembros de seguridad privada que interfieren con la libre apropiación del espacio por parte de los habitantes y visitantes; el derecho a la ciudad es, entonces, inexistente en tales sitios.

La decisión de las últimas administraciones públicas municipales y estatales de asentar en el sitio algunas instancias de gobierno ha contribuido a la consolidación de dicha zona del centro histórico como una centralidad (Vélez, 2007) gentrificada. Se encuentra latente la especulación inmobiliaria pues empresas internacionales ya han comprado predios e inmuebles en la zona para la creación de infraestructura de servicios. Desde la implementación del proyecto hasta la actualidad ha ocurrido un proceso de transformación de usos de suelo con creciente presencia de usos comerciales, del paisaje urbano en el cual contrasta la modernidad del Centro de Convenciones con viviendas depauperadas e incluso en ruinas a escasos metros de aquél y del tejido social fracturado por el desplazamiento de los habitantes de los barrios.

Todo ello denota una situación de elitización de la zona, esto es, de gentrificación, con el consiguiente desplazamiento de los habitantes que por generaciones habían vivido en la zona de impacto del proyecto. La transformación cualitativa señalada se expresa en los siguientes datos:

Entre 1993 y 2008, se observa la disminución del número de inmuebles con uso residencial, de $58 \%$ en 1993 , a $35.4 \%$ en 2008 ; la pérdida de actividades productivas o industriales, que se redujo de 4 a $0.7 \%$; en contraste con el aumento de actividades comerciales y de servicios, de $2 \mathrm{I}$ a $36 \%$, y el notable incremento de predios desocupados o baldíos, de 13 a $23.7 \%$ (Cabrera, 2014: 9). 
De ese modo observamos que la política pública ha propiciado la gentrificación y la consiguiente exclusión de los antiguos habitantes, situación que contrasta frontalmente con los planteamientos del "derecho a la ciudad".

\section{Conclusiones}

Tradicionalmente el Estado se ha apropiado del derecho de decidir sobre la ciudad y el territorio, bajo la idea de promover su ordenamiento y desarrollo, pero es justamente este orden el que se encuentra actualmente en cuestión, emergiendo la certeza de que los intereses privados del capital mueven la formulación y aplicación de la política pública, dejando de lado los intereses colectivos de los ciudadanos. Actualmente el propio término de derecho a la ciudad propuesto por Lefebvre se encuentra entre dos contenidos: el que retoman teóricos y activistas como un derecho colectivo para la autogestión de ciudades más justas, y el de los gobiernos y empresas que lo conciben sólo como un conjunto de garantías individuales que el Estado debe conferir a sus habitantes. A la par observamos el surgimiento de una ciudadanía que reclama su derecho a ser partícipe de la construcción de su ciudad por medio del cuestionamiento hacia las políticas públicas implementadas pero, también, a través de la elaboración de propuestas alternativas sobre cómo dicha ciudad debe construirse material y simbólicamente. Esto no se dio de forma fortuita, tuvo también un proceso en el que, a raíz de la forma en que se llevaron a cabo las políticas urbanas y de experimentar sus consecuencias, se ha comprendido que la ciudad se ha construido sobre la desigualdad, el despojo, la segregación y la violencia.

En el caso de Puebla, la fundación es ejemplo de que las desigualdades territoriales tienen parte de su origen en la manera en que se decide, desde el poder y bajo el interés de favorecer a una parte de la población en detrimento de otra. 
Con sus diferentes formas de llevarse a cabo a través de los años, esta expresión de la violencia en el territorio urbano persiste. Ejemplo de ello es la política urbana aplicada a finales del siglo xx en el mercado La Victoria y el proyecto del Paseo San Francisco. Con la finalidad de desconcentrar el centro histórico y dotar de infraestructura y servicios a la emergente urbanización, se consideró necesario, por parte de los grupos hegemónicos en alianza con el Gobierno, cerrar el mercado La Victoria para trasladar a los comerciantes a la Central de Abastos y a otros mercados de apoyo, varios de los cuales están actualmente deteriorados y parcialmente abandonados.

La respuesta por parte de las autoridades contra la resistencia de los comerciantes y ambulantes para abandonar el mercado es una muestra de que, de forma simbólica y física, la violencia es instrumentada para llevar a cabo una política urbana que puede ser en sí misma violenta. La dupla violencia-política urbana permea el proyecto Paseo San Francisco, pero en este caso afectando la forma de vida de los trabajadores y los habitantes del lugar, cuya resistencia no logró detener la aplicación de dicha política, pero en su transcurso la ciudadanía develó, con su palabra y acciones, el carácter violento de la política pública.

Bibliografía

Borja,J. (2002). Espacio público y derecho a la ciudad. Viento Sur, núm. II6, pp. 39-49.

-. (20।3). Revolución urbana y derechos ciudadanos. Madrid: Alianza Editorial.

Cabrera, L. (20।4). El proyecto del Paseo del Río San Francisco, Puebla, México. Gentrificación fallida, dirigida por el estado y la inversión privada. Coloquio Internacional Perspectivas del Estudio de la Gentrificación en México y Latinoamérica, I-4 de abril. Working paper series, Contested Cities. México: UnAM-Instituto de Geografía/Madrid: Universidad Autónoma de Madrid. 
Cabrera,V. (2008). Políticas de renovación en centros históBibliografía ricos de México. Centro H., núm. I, pp. 20-39.

García, R. (2008). Sistemas complejos. Conceptos, método y fundamentación epistemológica de la investigación interdisciplinaria. Barcelona, España: Gedisa.

Guerrero,J.(2005).Transformación urbana e inserción social y espacial en el centro de Puebla, México. En Bodson et al., Vivir en la ciudad. Lo cotidiano en la inserción urbana en México,América Central y el Caribe. San José: flAcso I.

Harvey, D. (2005). Breve historia del neoliberalismo. Madrid: Ediciones Akal.

- (20I2). Ciudades rebeldes. Del derecho a la ciudad a la revolución urbana. Madrid: Ediciones Akal.

Hasan, A. (20I0). El concepto de ciudad de clase mundial y sus repercusiones en la planificación urbana para las ciudades de la región de Asia Pacífico. En Sugranyes, Ana, y Mathivet, Charlotte, Ciudades para tod@s, por el derecho a la ciudad, propuestas y experiencias (pp. 303316). Chile: HIC.

Hernández, A. (20I5). Del límite a la revalorización del patrimonio: Barrio de San Antonio Puebla. On the W@ terfront, $40(2)$.

Hernández. (2016, julio 16). Operativo contra piratería desata balacera en Puebla; hay 15 heridos. Proceso. Recuperado de: https://www.proceso.com.mx/448320/ operativo-contra-pirateria-desata-balacera-en-puebla-15-heridos

INEGI. (2017). Encuesta Nacional de Victimización y Percepción sobre Seguridad Pública. Recuperado de: https://www. inegi.org.mx/programas/envipe/20I7/

Instituto Municipal de Planeación Puebla (Implan). (2019). Carta poblana por el derecho a la ciudad. Recuperado de http://pueblacapital.gob.mx/images/2019/Carta Poblana_por_el_derecho_a_la_ciudad_10-19.pdf 
Bibliografía

López Obrador, A. M. (2019). Discurso pronunciado en el "Foro Planeando Juntos la Transformación de México para Organizar el Plan de Desarrollo 2019-2024. aristeguinoticias.com

López, V., López, A., y Olvera, M. (2018). Ruptura disciplinar en el estudio del territorio y la inclusión de la ética. En Cabrera, V., y López, V., Ética y poder en la configuración territorial. Benemérita Universidad Autónoma de Puebla.

Marcuse, P. (2010). ¿Los derechos en las ciudades y el derecho a la ciudad? En Sugranyes, A., y Mathivet, C., Ciudades para tod@s, por el derecho a la ciudad, propuestas y experiencias (pp. 91-104). Chile: HIC.

Meléndez, M. (2017). Espacio público, actores urbanos y sus apropiaciones sociales: Un acercamiento antropológico a Paseo de San Francisco. Puebla: CAS BUAP.

Morin, E. (200I). El método. La naturaleza de la naturaleza. Madrid, España: Ediciones Cátedra.

- (2006). El método. El conocimiento del conocimiento. Madrid, España: Ediciones Cátedra.

Ornelas,J.(2004). Impacto de la globalización neoliberal en el ordenamiento urbano y territorial. Papeles de Población, núm. 4I, PP. I-24.

Patiño, E. (2002). El pasado en el presente: Pobreza, centro histórico y ciudad. Programa Editorial de la Red de Investigación Urbana. Puebla: UAP.

Pérez, M. (20I5). Ciudadanía urbana y derecho a la ciudad: Hacia una política del habitar. En: Gasic, Ivo, Narváez, Ángelo, y Quiroz, Rodolfo, Reapropiaciones de Henri Lefebvre: Crítica, espacio y sociedad urbana (pp. 10-30). Chile:Triángulo.

Revista Patrimonio Cultural. (1994). El plan Angelópolis y el Proyecto del Río San Francisco. Revista Patrimonio Cultural, núm. I, Pp. 19-29. Área de Estudios para la Conservación del Patrimonio Cultural. Puebla, México. 
Salazar, C. (2005). Formas de violencia en la Puebla del siglo Bibliografía XVII, a la luz de los anales del barrio de San Juan del Río. Historia y Antropología de Puebla, núm. 78, pp. 3-II.

Salazar, F. (2005). Globalización y política neoliberal en México. Cuadernos de Política en México, Pp. I-I I. México. Vélez, F. (2007). Planeación, crecimiento urbano y cambio social en el centro histórico de la ciudad de Puebla. Puebla: BUAP. Zizek, S. (2009). Sobre la violencia. Seis reflexiones marginales. Barcelona, España: Ediciones Paidós Ibérica. 